\title{
Collaborative use of Sixth Sense Device, Blue Brain and Swarm Intelligence for Performing Joint Actions
}

\author{
Rimmy Chuchra \\ Department of \\ Computer Science \& Engg \\ Sri Sai College of Engg. And Technology, \\ Manawala (Amritsar)Punjab
}

\author{
R.K. Seth \\ Department of Applied Science \\ Sri Sai University,Palampur
}

(H.P)

\begin{abstract}
Around the globe, scientists and researchers are discovering new methodologies and techniques to download the data stored in the brain of a dead person for its further utilization. After the clinical death of the body, the scientists would use intelligence of any person in the form of blue brain which is also called as virtual or artificial brain that works actively when inserted as robotics machine. The paper presents the synergetic interaction between man and machine having master-slave relationship where a man act as a master and nano-robot act as slave. The nano-robot uses sixth sense technology device for mind mapping utilizing blue brain with swarm intelligence. The benefit to utilize swarm intelligence is to provide social interactions with the environment and utilizing the blue brain benefits for restoration of knowledge, memory and past experiences of a person for the further development of society. The collaboration between man and machine intelligence leads to achieve a smart or intelligent joint action for developing projects in any industry or daily life works.
\end{abstract}

\section{Keywords}

Nano-robot, sixth sense, blue-brain, swarm intelligence.

\section{Index Terms:}

Man, Machine, Finite Intelligence and Infinite Intelligence.

\section{INTRODUCTION}

Artificial Intelligence (A.I) is a type of spiritual research [11] that covers a broad era of computer science and is responsible for the creation of intelligent methods and machines. The aim of A.I is to put intelligence into machines by utilizing different methods or strategies for solving complicated problems [28] so that machines may behave like humans [16]. It may consider a variety of data sets for solving problems different parameters. For solving any problem in A.I, the researchers always start from that specific problem along with planning, reasoning, learning, knowledge, processing and its perception etc [27]. The field of A.I has a wider scope into many different areas that may be listed below:

- In hospitals: A. I is used to store computer-aided information of medical images in the database.

- In Computer Science: A.I is used for Automatic Storage Management and Programming etc.

- In Heavy Industry: It is utilized in robotics for performing different types of tasks. For example; the teams of robots may be used for developing projects that may further relate to different fields for the use of robots for sensing environmental information. [29]
The use of artificial intelligence covers a broad era of technical world that may in future helps us to take better decision in our daily life problems that automatically improves the quality of life.

This paper presents a collaborative approach for utilizing intelligence by considering two separate applications of artificial intelligence viz. nano-robot having blue brain and sixth sense technology device that utilizes swarm intelligence and the function of each has been described separately as follows:

- Blue brain (BB) is a type of artificial brain or virtual brain that can act like a natural brain. The reason for considering blue brain is to utilize finite intelligence stored in the brain of a dead body[13]. The blue brain may be diagrammatically represented as shown in fig.1.

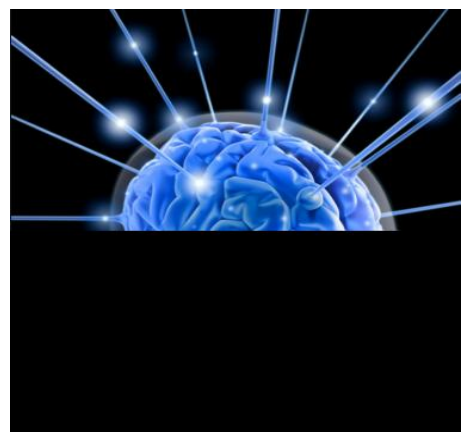

Fig 1: Blue Brain/Artificial Brain. [Accessed on 6/1/2016] [31]

- Nano-Robot (NR) is a type of tiny machine that is used for performing different types of tasks. The complete working of tiny machine depends on the type of algorithms and mechanisms programmed under [30] and may be diagrammatically represented as shown in fig.2:

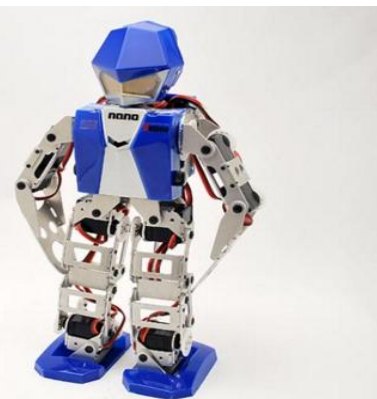

Fig 2: Nano-Robot-Tiny Machine. [Accessed on 6/1/2016] [32] 
- Swarm Intelligence (SI) is used to represent the collective behavior in the form of interactions with the environment [9] and may be diagrammatically represented as shown in fig.3:

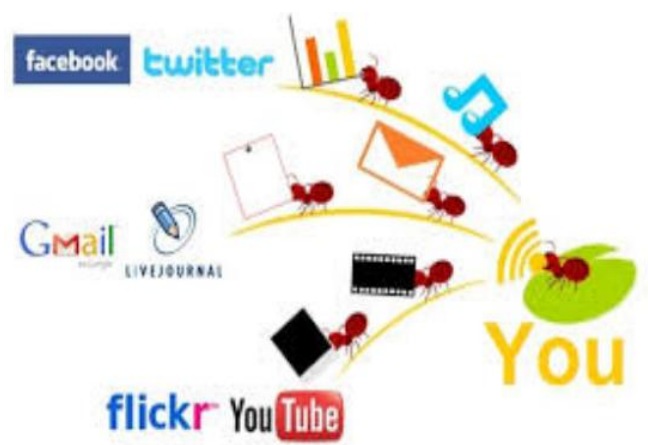

Fig 3: Use of Swarm Intelligence: Provides Social Interactions with the Environment. [Accessed on 6/1/2016] [33]

In addition, this naturally inspired global optimization technique has the capability of providing continues improvements for achieving more efficient results within short interval of time [4].

- Sixth sense Technology (SST) is a science of tomorrow having an aim of connecting the digital world into physical world seamlessly [14] that may be diagrammatically represented as shown in fig.4:

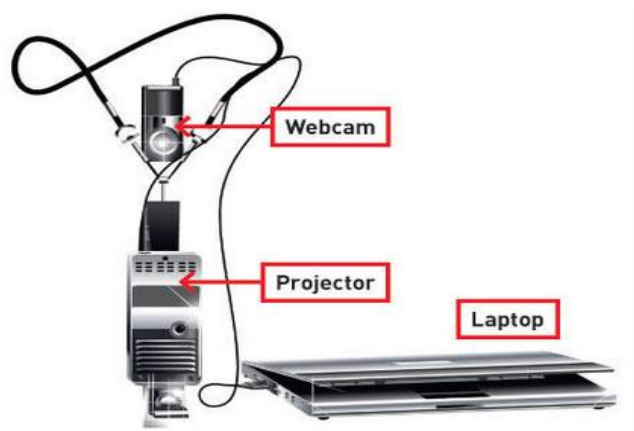

Fig.4: Sixth Sense Technology Device (SSTD). [Accessed on $6 / 1 / 2016]$ [34]

Sixth Sense Technology Device (SSTD) is a combination of five basic components such as camera, microphone, projector, mirror, color marker [21] and its working is based on three different types of technologies or behavior such as gesture recognition, image capturing and augmented reality etc. [18]. The main purpose to utilize this A.I application is to integrate the information and technology into an interactive manner so that both may be used in future efficiently for performing daily life tasks that automatically improve the quality standards of our life [17], [22]. Sometimes, sixth sense technology device is also called TED (Technology Entertainment Device) [23] where the working of TED depends on the type of software used [11]. It facilitates the people by changing the way of interaction medium with the digital device and human behavior as well [12], [24]. People utilizes transparent user interface(TUI) for accessing information about everything around us [20] and correspondingly it also helps to covers the gap between digital world and physical world [15] that may be diagrammatically represented in fig.5:

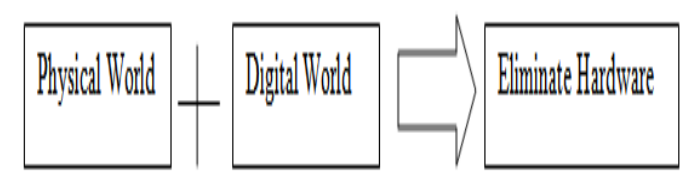

Fig 5: A Combination of physical \& Digital world: Eliminate (Reduce) Hardware.

On the other hand, it also helps to move towards a step that eliminates the need of hardware in the form of various digital devices such as mobile, PDA (Personnel Digital Assistant) and laptop in future [19]. The drawback of this technology is that the radiations emitted from the smart phone (a part of sixth sense technology device) harms human body by the emitted radiations that burn human body cells or tissues due to its use for a long span of time [10]. Having strong advantage because of the practical use of sixth sense technology device, the technology is being used in many different areas that may be discussed as follows.

- It provides innovative teaching by utilizing online media during delivering of lecture in the class room that ultimately eliminate the use of board and marker and increase the use of natural hand gestures movements [25].

- It provides interactive advertisements in newspapers in multimedia applications [26].

With the current innovative trends towards technology that automatically changes the way of interaction between man and machine. Such changes also show the reflections on man and machine relationship [24] by showing merged behavior that may be diagrammatically represented as shown in figure 6 \& figure 7. Fig. 6 shows a simple man and machine interaction.

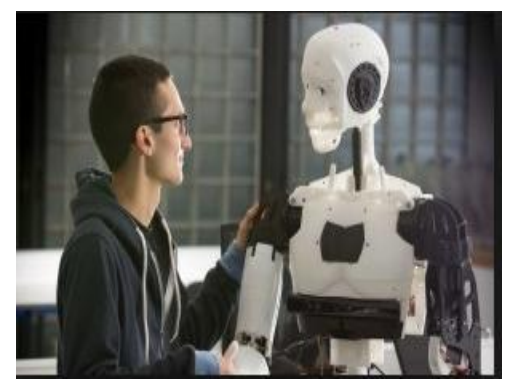

Fig 6: Interaction Medium shows : A Collaborative action is taken by man and machine.[Accessed on 06/01/2016][35]

Fig. 7 shows machine and machine interaction. 


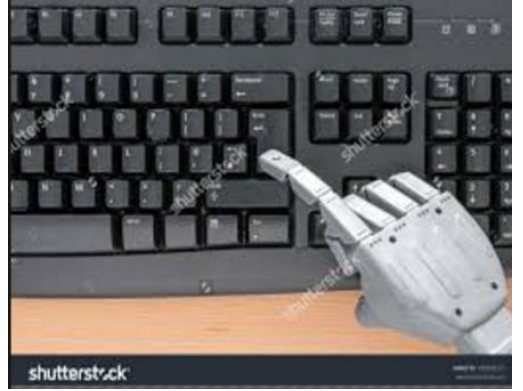

Fig 7:Machine-To-MachineInteraction Medium.[Accessed on $06 / 01 / 2016][36]$.

Utilizing two separate types of interactions collectively, the researchers try to develop a smart or intelligent joint action. For developing more intelligent methods to achieve more efficient results, the authors contribute their efforts for creating intelligent methods by merging two different applications of artificial intelligence viz. nano-robotics having blue brain and sixth sense technology device with swarm intelligence. Swarm intelligence for providing local interactions with the environment is also discussed. The benefit to use this proposed methodology is to develop an intelligent or smart joint action while providing social interactions with the environment during mind mapping by nano-robot that may be diagrammatically represented as shown in fig. 8 .

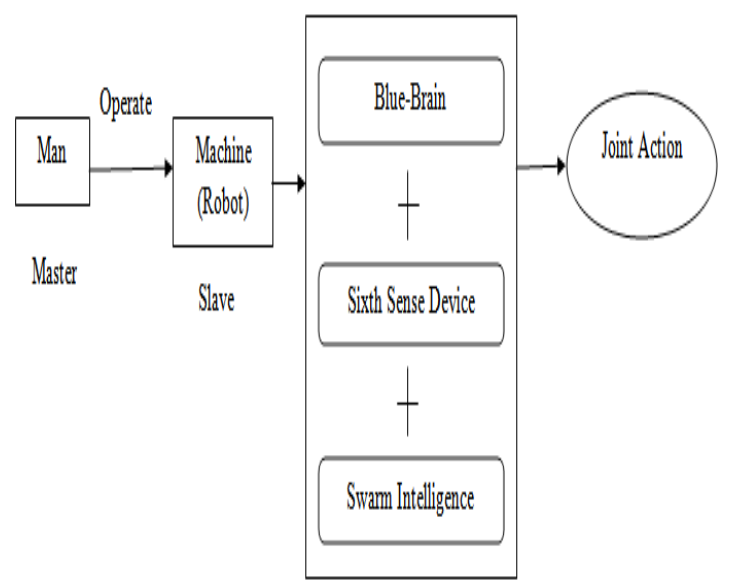

Fig 8: Joint Intelligent Action: Taken by Man \& Machine Collectively.

The procedure followed by authors in new designed methodology comprises nano-robot wearing sixth sense technology device for mind mapping of idea by utilizing blue brain after the death of the body. The working of new designed methodology is started when researchers inserted blue brain on nano-robot correspondingly nano-robot wears sixth sense technology device that gives output in the form of swarm intelligence (i.e. or in the form of interactions) through social interactions. The working of swarm intelligence is totally depends on the type of algorithms used by them. There are variety of algorithms that are used as like ant colony optimization, particle swarm intelligence, population based immune swarm intelligence algorithm, differential evolution swarm intelligence[5] etc. This paper uses brain storm optimization for solving separate types of problems [6].
After following this new designed methodology, it enters into a higher level of intelligence that further used for solving complicated problems. Operating multiple agents (robots) at a single time without centralized control is a classic example of the technology [7]. The motivation towards merging of two different A.I applications is: the utilization of natural and artificial intelligence simultaneously and diagrammatically represented as shown in fig.9.

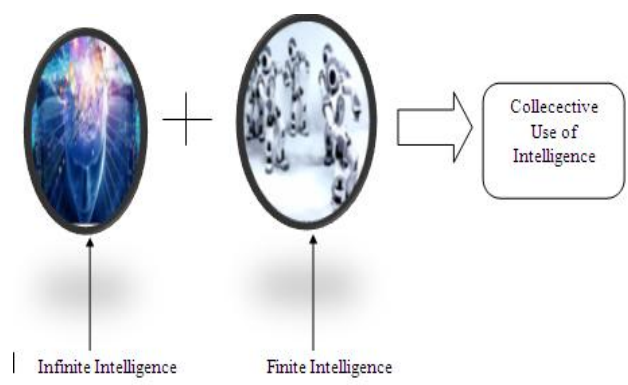

Fig 9: Combined Use of Natural \& Artificial Intelligence.

\section{II REVIEW OF LITERATURE}

(Chuchra et al. Feb-2013): This paper discussed about the joint action taken by the humans and robotics for performing the task. Humans and robotics hold master/slave relationship where interface acts as a sandwich between the humans and robotics. The use of interface is to provide communication between human and robotics in a natural way where human act as a master and is responsible for giving instructions to robotics and robotics always acts as a slave that work as per instructions given by their master. The communication flow methodology between human and robotics may be shown in fig.10 [2].

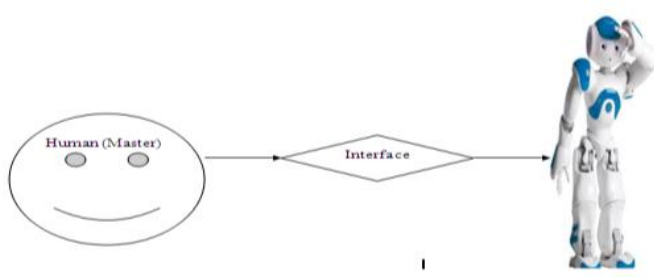

Fig 10: HRI: Shows Master/Slave Relationship.

(Seth \& Chuchra et al. May-June 2014): Authors had proposed a new procedure termed as "Human-robotics Interaction based analysis-Using data mining techniques" that shows how humans and robotics performed task collectively utilizing three different data mining techniques viz. classification, regression analysis and time series analysis. This designed methodology worked only with single type of data input format. It must be in discontinuous form (i.e. in digital form). Theoretical and experimental based analysis investigated in this paper with the outcome of significant results [1].

(Seth \& Chuchra et al. Jan 2015): Authors had designed a new communication flow methodology that works on two separate types of inputs given by the user viz. analog and digital form. The designed methodology also provides more natural way of communication while utilizing interface. Instructions can be easily managed by robotics given by the master-human [3]. 


\section{RESEARCH DESIGN}

A. Overview of Architectural Design: The communication flow methodology followed by a man shows interfaceinterface Interaction that can be diagrammatically represented as shown in fig. 11 .

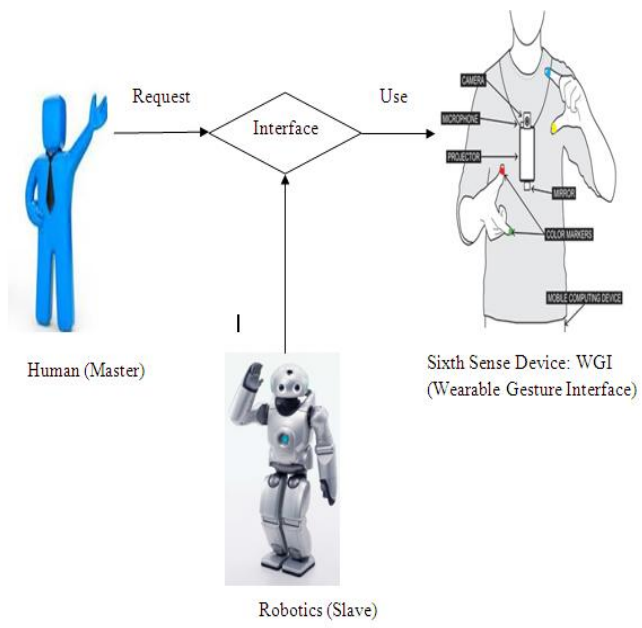

Fig 11: MIII: - Man-Interface-Interface-Interaction.

B. Detailed Architectural Design: The diagrammatical representation for taking an intelligent joint action and can be diagrammatically represented as shown in fig. 12 .
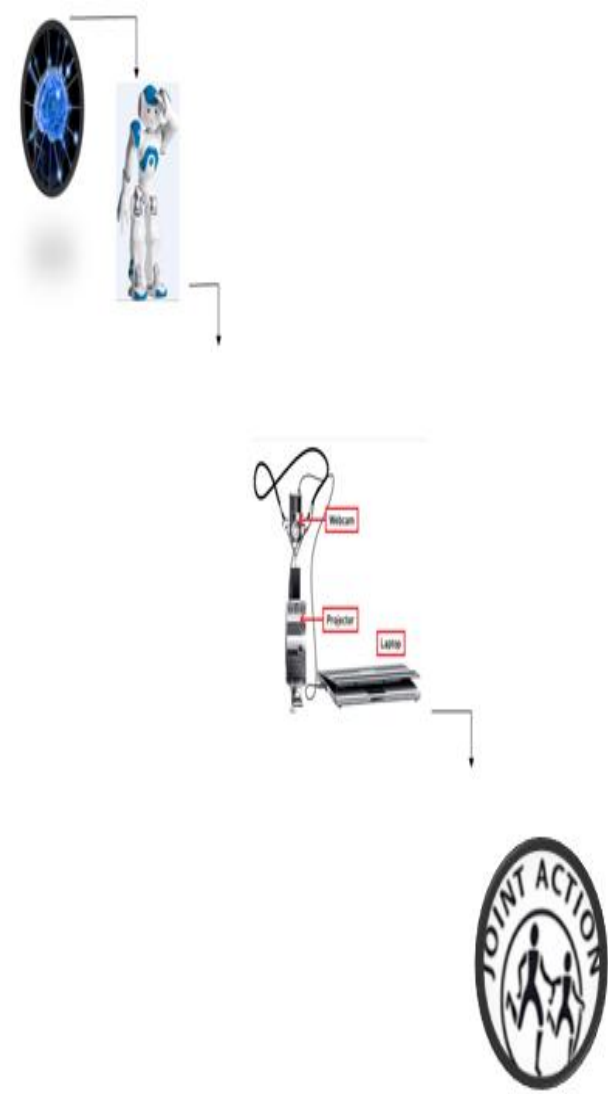

Fig 12: Man \& Machine: Shows Intelligent Joint Action.

\section{COMMUNICATION FLOW METHODOLOGY}

The communication between man and machine can be diagrammatically represented as shown in fig. 13 .

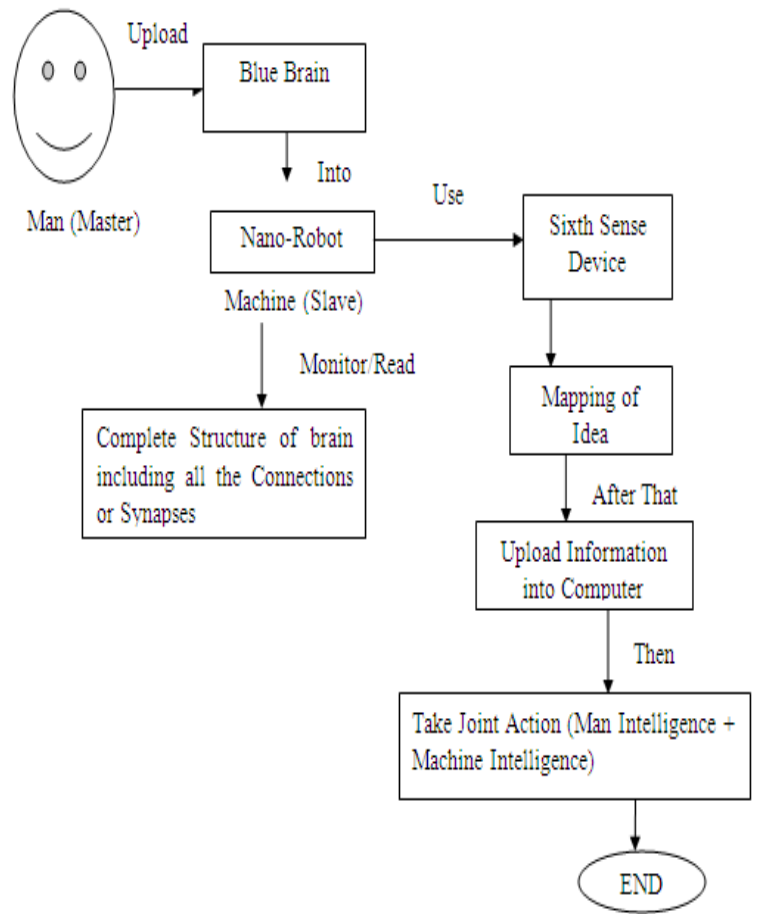

Fig 13: Communication Flow Methodology: Shows the way of interaction between man and machine.

\section{WORKING}

At first, the master (human) uploads blue brain into nanorobot that would act as a slave. The primary function of nanorobot is to understand the complete structure of brain including all the connections or synapses of the brain. In the second step, as per instructions given by his master, nanorobot uses own blue brain for utilizing finite intelligence and correspondingly wears sixth sense technology device for mind mapping of idea through social interactions with environment. This idea shaped by nano-robot that is either based on knowledge and past experiences that may either originate from the blue brain or observed from environment by utilizing the concept of swarm intelligence. In the third step, mapped idea or mapped information is simply uploaded on computer by master (human) after information collected from the slave (nano-robot) using some algorithms and mechanisms for performing some specific task. In this way, the researchers collaborate finite and infinite intelligence for developing a smart and intelligent joint action.

\section{CONCLUSIONS}

In this paper, the authors have merged two separate applications of artificial intelligence. viz. sixth sense technology device and nano-robot. The benefit for combining two separate applications is to study the collective behavior of a man and machine for proposing a new communication flow methodology that shows a collective use of man intelligence and machine intelligence while utilizing master-slave relationship. The proposed communication flow methodology 
as developed in this paper is named as Man-InterfaceInterface Interactions (MIII). The goal of MIII is to improve the level of collaborative learning utilizing man and machine intelligence. Ideas can be easily mapped by a nano-robot using sixth sense technology device and blue brain. It also helps to protect the human body from the exposure of radiations emitted from the sixth sense technology. In this way, the co-ordination between the man and machine leads to develop a smart and intelligent joint action for taking better decisions in life that ultimately improves our quality of life.

\section{REFERENCES}

[1] Rimmy Chuchra \& R.K Seth March-2014. Human robotics interaction based analysis-Using DMT, International Journal of Computer Science and Information Technology.

[2] Rimmy chuchra \& Ramandeep kaur, Feb 2013. Human robotics interaction with data mining techniques", International Journal of Emerging Technology and Advance Engg.

[3] R.K Seth \& Rimmy Chuchra Jan-2015. Synergetic Interaction among Humans and Robotics by Proposing Communication Flow Methodology, International Journal of Computer Applications.

[4] Soumya ghosal,Rajkumar darbar,Biswarup Neogi,Achintya das \& Dewaki N.Tibarewala, 2012.Application of swarm intelligence Computation Techniques in PID controller tunning: A Review, Proceeding of the Incon,Springer-Verleg.

[5] Hua bai, 2006. A Survey on application of swarm intelligence computation to electric power system IEEE.

[6] http://www.igi-global.com/calls-for-papersspecial/international -journal-swarm-intelligenceresearch/1149.

[7] P.Amudha \& Abdule H Rauf,Nov-2012: A study on swarm intelligence Techniques IN Intrusion Detection, International Journal of Computer Applications.

[8] Lars P. Linden, March-2008. A tool for Identifying swarm intelligence on a free open source software mailing list, University of Central Florida: Proceeding of the southern association for information systems Conference.

[9] Ramesh B Lakkakula,D Swampna,RVSN Sree RAMYA \& Kaja Mahetha, Feburay-2014. Artificial AcumenSwarm Intelligence: Proceeding of $3^{\text {rd }}$ IRF International Conference Chennai.

[10] www.slideshare.net/atinav technology-complete-ppt.

242/the-sixth-sense-

[11] www.ukessays.com/essays/media/sixth-senseTechnology-merging-real-and-virtual-worlds-mediaessay.php.

[12] Dheeraj Chandra Murai \& Vinod Kumar Verma,November-2014.Application of sixth sense technology in Lecture Theater for Engg. Students, International Journal of computer applications.

[13] Siva Kumar Avula, Vedrucha S.Pakale and Sheetal V Kashid March-2013. Blue Brain-The Future Generation,International Journal of application and innovation in Engginering and Management.
[14] www.spirtualresearchfoundation.org/spirtualresearch/sixth-sense/how-to-test-your-sixth-sense/.

[15] Jash Mehta, Nirav Nayani, 2014. A Review paper on Sixth Sense Technology, IN PRESSCO, International Press Co-corporation, INPRESSCO.

[16] Mrs Rekha Purohit, Sneh lata Vyas,June-2013.Artificial Intelligence and its application: Sixth Sense Technology, International Journal of Emerging Trends and Technologies in Computer Science.

[17] reloadybtech.blogspot.in/2012/01/sixth-senseTechnology.html.

[18] www.technologers.com/2013/06/pranav_mistry.html.

[19] theviewspaper.co.use/Sixth-sense-Technology/.

[20] R.Bavithra and L.Ayeesha Begame,May-2014.Mobile Projectors using sixth sense technology, Proceedings of $6^{\text {th }}$ IRF International conference, Chennai.

[21] techliebe.com/sixth-sense-technology/.

[22] Abinav Sharma,Mukesh Aggarwal,April-2013.Sixth sense Technology, International Journal on recent and innovation trends in computing and communication.

[23] www.cnet.com/news/mits-6th-sense-device-could-trumpapples-multitouch/.

[24] www.joout.com/sixth-sense-technology-4423789/.

[25] Meenakshi Gupta \& Shruti Sharma, October2014.Virtual class room using sixth sense technology, IOSR,Journal of Computer Science and Engg.

[26] Ms. Uttama.Suryanshi,Novemer-2013.How we look at the world forever by sixth sense technology, International Journal of Advanced research in Computer Science and software engg.

[27] http://en.wikipedia.org/wiki/Artificial_intelligence.

[28] https://medium.com/@ shivin/the-current-state-ofmachine-intelligence-f $76 \mathrm{c} 20 \mathrm{db} 2 \mathrm{Fe} /-$.

[29] http://en.wikipedia.org/wiki/Applications_of_artificial_in telligence.

[30] http://whatis.techtarget.com/definition/nanorobot.

[31] https://www.google.co.in/search?hl=en\&site=imghp\&tb $\mathrm{m}=\mathrm{isch} \&$ source $=\mathrm{hp} \& \mathrm{biw}=1366 \& \mathrm{bih}=623 \& \mathrm{q}=$ blue + brain $\&$ oq=blue+brain\&gs_l=img.3...1112.3391.0.3644.12.9.0. 0.0.0.463.463.41.1.0...0...1ac.1.64.img..11.1.463.ssvPvA hJV90\#imgrc $=$ dhDkV_H3d18U7M\%3A.

[32] https://www.google.co.in/search?hl=en\&site=imghp\&tb $\mathrm{m}=\mathrm{isch} \&$ source $=\mathrm{hp} \& \mathrm{biw}=1366 \& \mathrm{bih}=623 \& \mathrm{q}=$ nanorobot\&oq=nanorobot\&gs_l=img.3...2027.3984.0.4233.10.8.0.0.0.0.443.4 43.41.1.0...0...1ac.1.64.img..9.1.443.0HHRsmNtFo\#hl=e n\&tbm=isch\&q=nanorobot\&imgrc $=X-$ u6sFkfDAyxSM\%3A

[33] https://www.google.co.in/search?hl=en\&site=imghp\&tb $\mathrm{m}=\mathrm{isch} \&$ source $=\mathrm{hp} \& \mathrm{biw}=1366 \& \mathrm{bih}=623 \& \mathrm{q}=$ nanorobot\&oq=nanorobot\&gs_l=img.3...2027.3984.0.4233.10.8.0.0.0.0.443.4 43.41.1.0...0...1ac.1.64.img..9.1.443.0HHRsmNtFo\#hl=e $\mathrm{n} \& \mathrm{tbm}=\mathrm{isch} \& \mathrm{q}=\mathrm{swarm}+$ intelligence + social+interaction \&imgrc $=$ jpwBzw86bWnsAM\%3A 
[34] https://www.google.co.in/search?hl=en\&site=imghp\&tb $\mathrm{m}=$ isch \&source $=\mathrm{hp} \& \mathrm{biw}=1366 \& \mathrm{bih}=623 \& \mathrm{q}=$ nanorobot\&oq=nano-

robot\&gs_l=img.3...2027.3984.0.4233.10.8.0.0.0.0.443.4 43.41.1.0...0...1ac.1.64.img..9.1.443.0HHRsmNtFo\#hl=e $\mathrm{n} \& \mathrm{tbm}=\mathrm{isch} \& \mathrm{q}=$ sixth+sense+technology+devics\&imgrc $=$ nnCivho-BbAiQM\%3A

[35] https://www.google.co.in/search?hl=en\&site=imghp\&tb $\mathrm{m}=$ isch \&source $=\mathrm{hp} \& \mathrm{biw}=1366 \& \mathrm{bih}=623 \& \mathrm{q}=$ nanorobot\&oq=nano-

robot\&gs_l=img.3...2027.3984.0.4233.10.8.0.0.0.0.443.4 43.41.1.0...0...1ac.1.64.img..9.1.443.0HHRsmNtFo\#hl=e $\mathrm{n} \& \mathrm{tbm}=\mathrm{isch} \& \mathrm{q}=$ machine + and + machine + interaction $+\mathrm{rob}$ o+with+keyboard\&imgrc=QeJN-F5s7fyiM\%3A.

[36] https://www.google.co.in/search?hl=en\&site=imghp\&tb $\mathrm{m}=\mathrm{isch} \&$ source $=\mathrm{hp} \& \mathrm{biw}=1366 \& \mathrm{bih}=623 \& \mathrm{q}=$ nanorobot\&oq=nano-

robot\&gs_l=img.3...2027.3984.0.4233.10.8.0.0.0.0.443.4 43.41.1.0.......1ac.1.64.img..9.1.443.0HHRsmNtFo\#hl=e $\mathrm{n} \&$ tbm $=\mathrm{isch} \& \mathrm{q}=$ robot + use + keyboard \&imgrc $=7 \mathrm{oBt} 6 \mathrm{VN}$ 7XryPrM\%3A. 